\section{El infarto oculto. Las claves para el diagnóstico precoz de infarto posterior}

\author{
FERNANDO PINEDA ${ }^{1,2}$, BRUNO DIGHERO ${ }^{3}$, JORGE MERUANE $^{3}$, \\ PABLA CATALDO ${ }^{2}$, POLENTZI URIARTE $^{2}$
}

\section{True posterior infarction diagnosis. Report of three cases}

Acute Coronary Syndrome with ST segment elevation requires rapid reperfusion, which is why the timely identification of these conditions, based on the patient's symptoms and the correct interpretation of the EKG, is essential for therapeutic decisions. We report three patients with True Posterior Infarction and their difficult EKG diagnosis. The classic 12-lead EKG method is often not capable of supporting the diagnosis, so we recommend the use of posterior leads and mirror images observing inverted V1-V2-V3 leads on the classic EKG.

(Rev Med Chile 2021; 149: 1223-1230)

Key words: Acute Coronary Syndrome; Electrocardiography; Myocardial Infarction; Reperfusion.

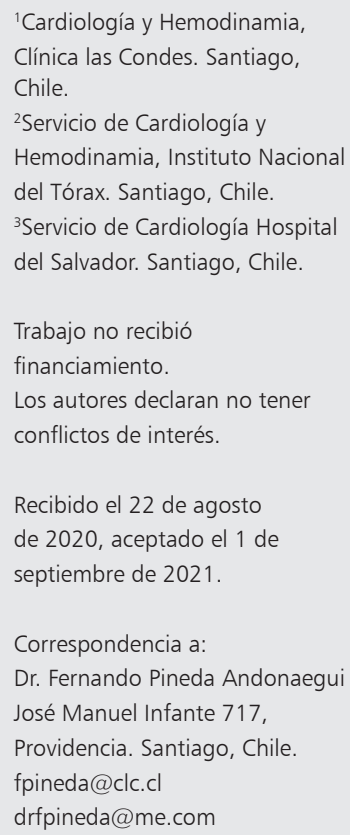

$\mathrm{P}$ or síndrome coronario agudo se entiende a la rotura de una placa ateroesclerótica en una arteria coronaria sobre la que se forma un trombo; de la cuantía del compromiso del flujo coronario que produzca este trombo va depender la severidad y gravedad del cuadro. Así, la obstrucción incompleta originará isquemia subendocárdica. En cambio, la oclusión completa del lumen arterial producirá isquemia transmural. En este último caso, de no recuperarase precozmente el flujo coronario, se producirá una significativa necrosis miocárdica. La distinción entre ambos cuadros la dará en electrocardiograma (ECG): en el primer caso se manifestará como síndrome coronario agudo sin supra desnivel del segmento del ST (SCAsSDST) y en el segundo caso como síndrome coronario agudo con supra desnivel del segmento ST (SCAcSDST), lo que requiere de una rápida reperfusión, ya sea por uso de la trombolisis (eliminación del trombo por método farmacológico) o de la angioplastía primaria (método mecánico) ${ }^{1}$.

Es por esto que la identificación oportuna de los cuadros de SCAcSDST, basándose en la clínica del paciente y la correcta interpretación del ECG, son fundamentales a la hora de decidir el tratamiento.

El compromiso de la pared posterior llamada por algunos investigadores "dead angle infarction" está asociado a pared inferior y/o lateral en 15 a $21 \%$ de los casos 2 , pero sólo 3,3 a 11\% se presenta como compromiso de pared única ${ }^{3,4}$. En un registro de más de 2 millones de infartos en Estados Unidos de Norteamérica, entre los años 2007 a 2014 el 1\% correspondió al llamado infarto posterior verdadero (IPV); sin embargo, el IPV presentaba un mayor riesgo de mortalidad intrahospitalaria ${ }^{5}$.

El infarto de pared posterior fue inicialmente identificado en el ECG como Rs en V1-V2 por Perloff $1964^{6}$. Esta descripción ha sido posteriormente cuestionada, adjudicándose ${ }^{7}$ a la pared lateral como la verdadera responsable del fenómeno electrocardiográfico mencionado.

Bayes de Luna propone una nueva terminología para esta pared, basada en la correlación con resonancia magnética cardiaca y reclasifica los infartos de la zona inferolateral, dividiéndolos en 3 grupos: inferior (Q en II, III y VF), lateral (RS V1 y/o Q patológica en derivaciones laterales) e inferolateral (ambos patrones) ${ }^{8}$. 
Independiente de la nomenclatura que se utilice, su diagnóstico por ECG es complejo y muchas veces se interpreta como SCAsSDST con las consecuencias que esto trae.

Todos los casos clínicos presentados cuentan con los consentimientos informados, autorizados por los pacientes y la evaluación de comité de ética del área Oriente.

A continuación presentamos tres casos de pacientes cursando IPV y su difícil diagnóstico por medio del ECG.

\section{Caso 1}

Paciente femenina de 31 años, sin antecedentes cardiovasculares, que inicia dolor súbito retroesternal en reposo, EVA 9/10, asociado a disnea y diaforesis. Al examen físico: PA 150/90 mmHg, pulso $74 \mathrm{lpm}$ regular, afebril, con examen cardiopulmonar sin alteraciones.

Se realiza ECG clásico de 12 derivaciones (Figura 1).

Por sospecha de SCAcSDST de pared posterior se solicita coronariografía (Figura 2).
La paciente evoluciona sin angor posterior a la angioplastía, con curva enzimática compatible con SCAcSDST. Ecocardiograma evidenció hipocinesia del segmento basal lateral (pared posterior).

\section{Caso 2}

Paciente mujer de 79 años sin factores de riesgo cardiovascular conocidos, que inicia cuadro de dolor retroesternal opresivo, EVA 10/10, asociado a disnea en reposo y sudoración profusa. Al examen físico PA: 120/50 mmHg, pulso: $114 \mathrm{lpm}$, sudorosa. Su examen cardiopulmonar resultó dentro de límites normales.

Se realiza ECG clásico de 12 derivaciones y derivadas posteriores (Figura 3 ).

Frente a la sospecha de estar frente a un SCAcSDST de pared posterior se llevó a cabo estudio hemodinámico con coronariografía de urgencia (Figura 4).

Se practica angioplastía, posterior a la cual la paciente evoluciona sin angor, con curva enzimática compatible con SCAcSDST.

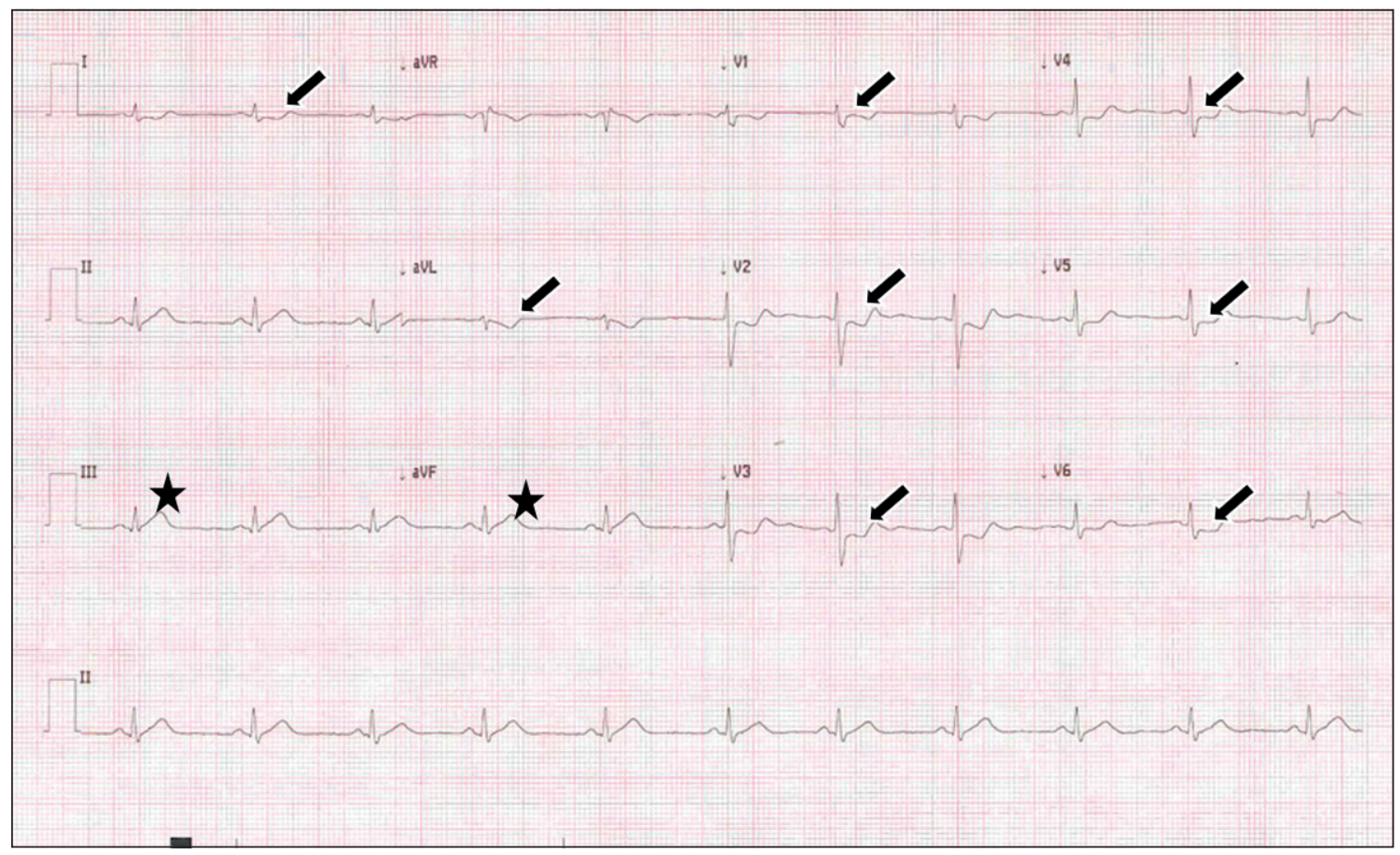

Figura 1. Caso 1: ECG: Ritmo sinusal $66 x$ ', conducción AV e IV normal. Se observa leve elevación del punto "J" en D3 y $\operatorname{aVF}(\star)$, e infradesnivel de ST en D1, aVL y precordiales $V 1$ a $V 6(\boldsymbol{\Lambda})$. 

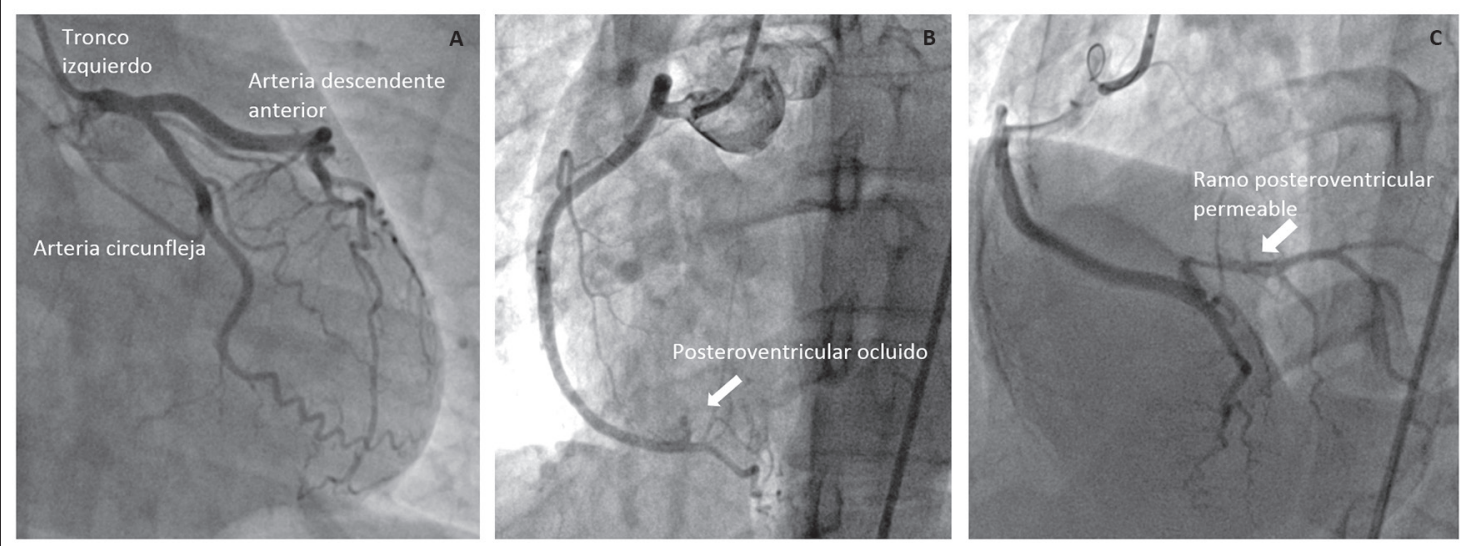

Figura 2. Caso 1: A-B: Coronariografía: tronco izquierdo, arteria descendente anterior y arteria circunfleja sin lesiones. Oclusión proximal del ramo posteroventricular de la arteria coronaria derecha. C: Angioplastía primaria al ramo posteroventricular de la arteria coronaria derecha.

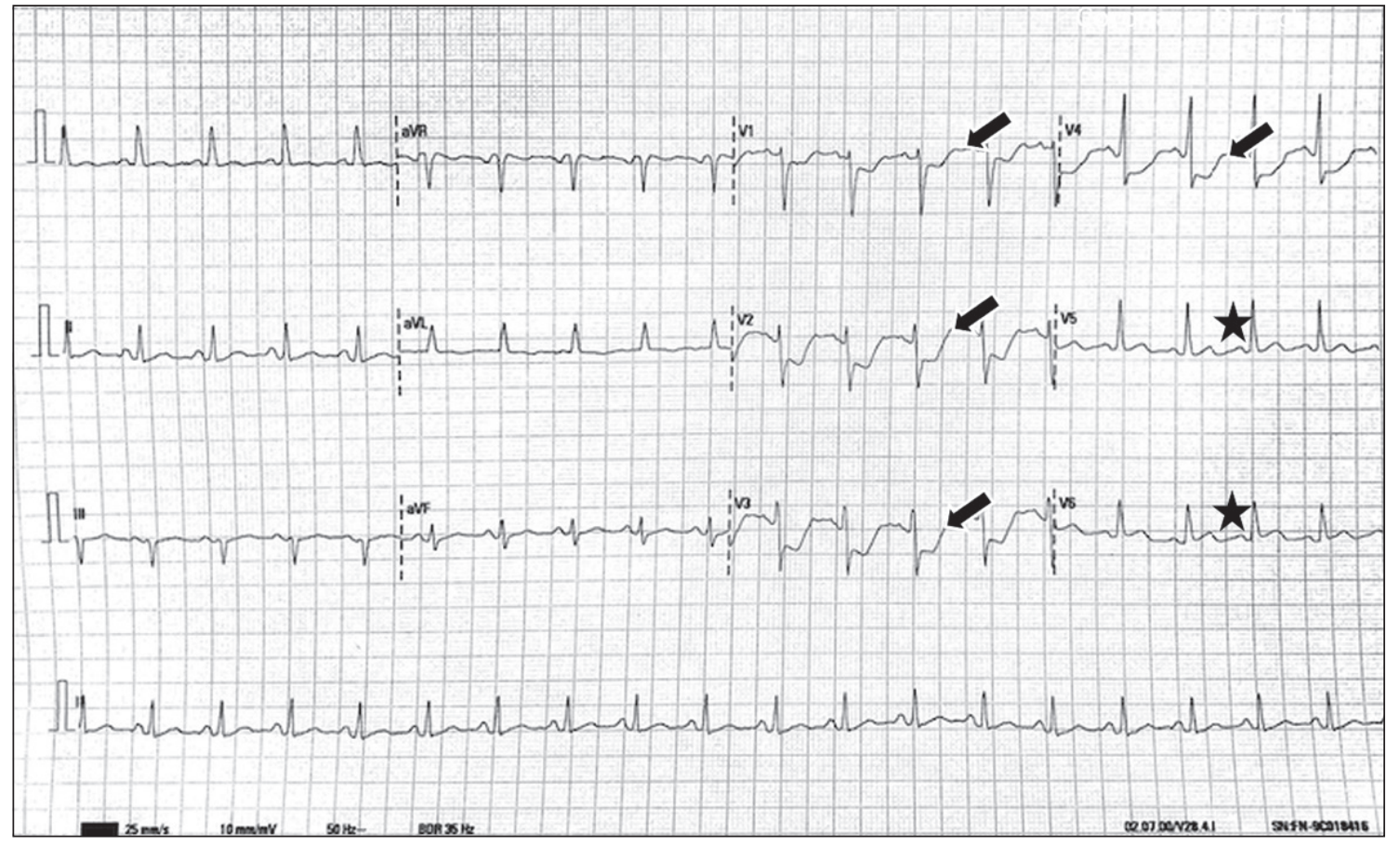

Figura 3A. Caso 2: ECG: Ritmo sinusal 116x, conducción AV e IV normal. Se observa mínimo SDST en V5V6 ( $\star$ ) y acentuado infradesnivel de ST de V1 a V4 ( $/$ ); no se observan cambios en la cara inferior significativos, como tampoco alteraciones de ST en aVR (compromiso proximal de la A. descendente anterior, o compromiso de tronco). 


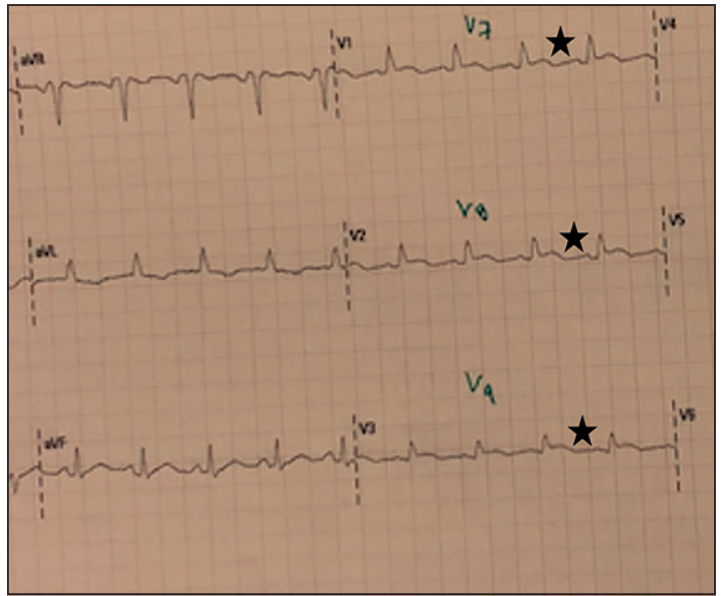

Figura 3B. Caso 2: En las derivadas posteriores V7 V8 V9, se observa discreto SDST $(\star)$.

\section{Caso 3}

Mujer de 74 años. Antecedentes de hipertensión arterial, dislipidemia, tabaquismo activo. Ingresa por un primer episodio de dolor retroesternal opresivo EVA 8/10 y síntomas. Al examen físico: PA 130/95 mmHg, pulso: $84 \mathrm{lpm}$, sin apremio ventilatorio, su examen cardiopulmonar es normal.

Se adjunta EEG clásico 12 derivaciones (Figura 5).

Frente a la sospecha de estar frente a un SDST posterior, se llevó a cabo estudio hemodinámico con coronariografía de urgencia (Figura 6).

La paciente evolucionó en buenas condiciones, desarrollando curva enzimática característica de SCAcSDST.

\section{Discusión}

La importancia de la rápida reperfusión en el infarto agudo al miocardio con SDST radica en una menor mortalidad y menos complicaciones cardiovasculares a corto y largo plazo ${ }^{9,10}$.

Específicamente en el infarto de pared posterior, el compromiso trombótico de la arteria circunfleja o ramo postero-ventricular (como se observó en el primer caso), es definido como el segmento infra-atrial del ventrículo izquierdo por debajo del surco atrioventricular. Su precoz tratamiento se asocia a disminución de la mortalidad y complicaciones como necrosis del músculo papilar posterolateral. En $22 \%$ se asocian a insuficiencia mitral moderada a severa ${ }^{11}$, sobretodo en presencia de dominancia izquierda ( $8 \%$ de la población $)^{12}$.

Como se presentó en los casos clínicos de estos 3 pacientes con clínica de SCA, las alteraciones clásicas de SDST en el ECG de 12 derivadas están ausentes $y$, por ende, la oportunidad de una rápida reperfusión puede no lograrse.

En algunas oportunidades se puede observar rotación del eje del corazón por lo que la necrosis de la parte basal lateral, clásicamente llamada pared posterior, no puede generar onda "Q" en las derivaciones posteriores ni onda " $R$ " en $\mathrm{V} 1$ debido a que esa zona se despolariza con un retraso de $40 \mathrm{~ms}^{13}$.

La derivada que registra la actividad eléctrica de la zona opuesta a la zona en evaluación se denomina "derivada recíproca" y, muchas veces, puede entregar importante información;

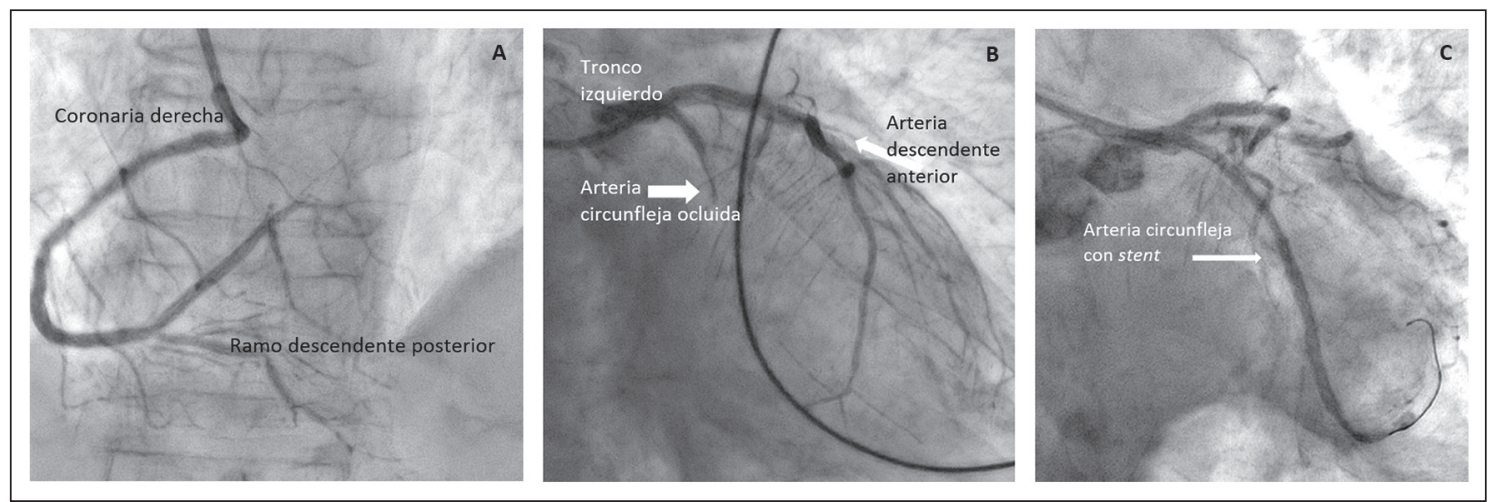

Figura 4. Caso 2: A-B: Coronariografía de urgencia: arteria coronaria derecha sin lesiones destacables, ramo descendente posterior con enfermedad severa crónica. Tronco izquierdo sin lesiones. Arteria descendente anterior con algunas irregularidades, arteria circunfleja con oclusión total proximal. C: Angioplastía primaria con stent a la arteria circunfleja proximal. 


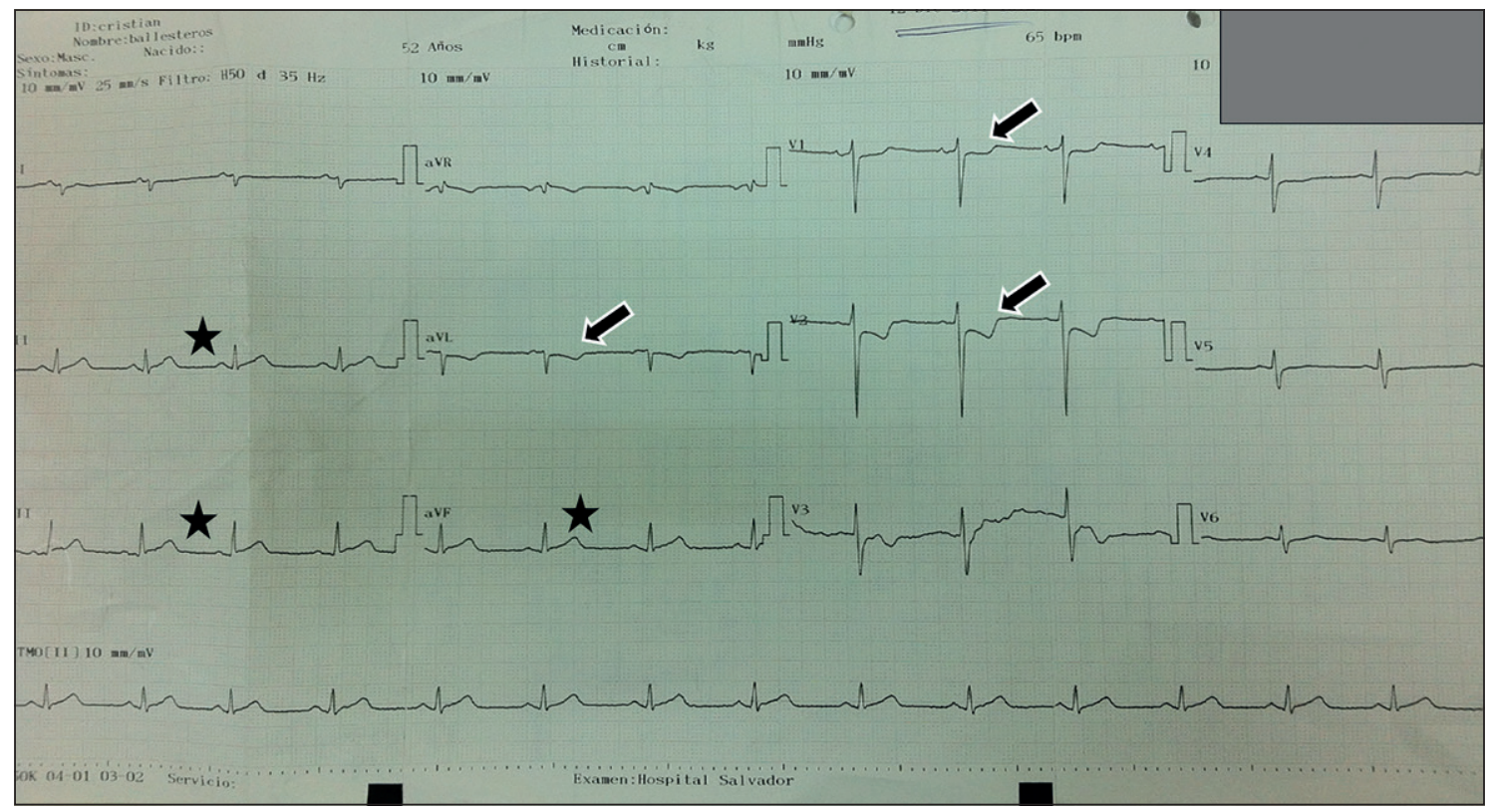

Figura 5. Caso 3: ECG: Ritmo sinusal 84 x`, conducción AV e IV normal, aparece mínimo SDST en cara inferior D2 D3 aVF $(\star)$ e infradesnivel de ST en aVL y precordiales derechas $(\boldsymbol{\Lambda})$, en asociación a un eje eléctrico de QRS a derecha. Esto puede interpretarse como infarto de pared inferior con compromiso de la "pared posterior".
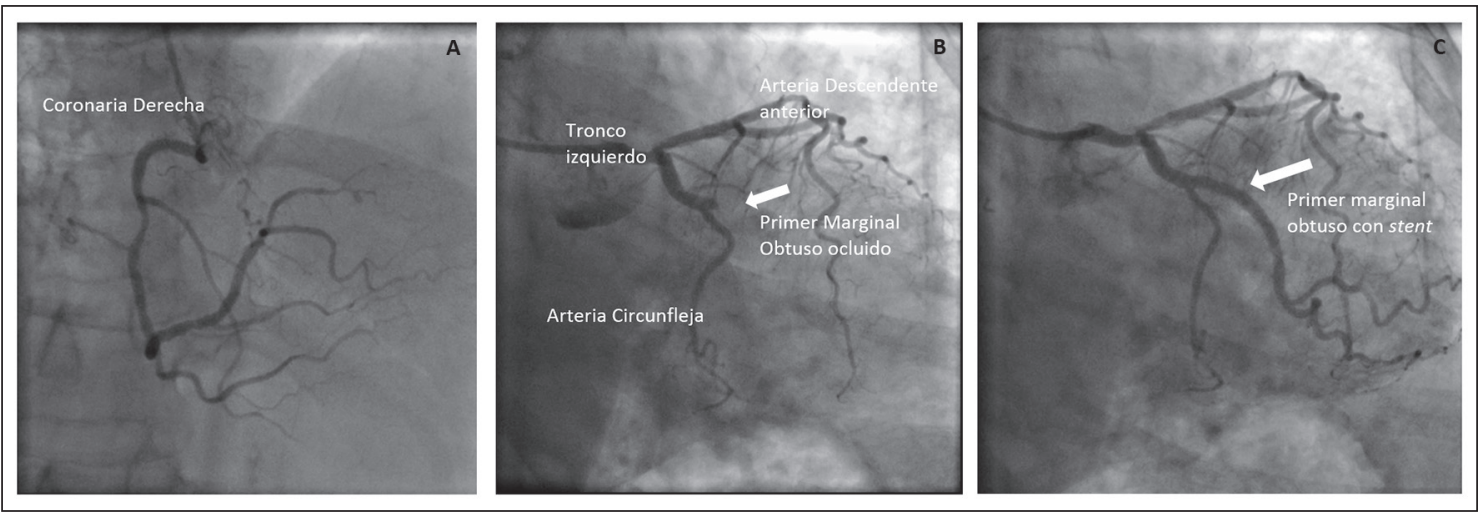

Figura 6. Caso 3: A-B: Coronariografía: arteria coronaria derecha con placa moderada en tercio medio. Tronco común izquierdo con placa leve distal, arteria descendente anterior con placa severa en segmento medio. Arteria circunfleja con irregularidades. Primera marginal obtusa ocluida en su inicio. C: Angioplastía primaria con stent al primer marginal obtuso proximal. ECG clásico 12 derivadas, imagen especular de forma invertida, la flecha indica el SDST.

las derivadas reciprocas de la pared posterior en el ECG son V1-V2. Una depresión del ST en V1 y V2 puede ser la imagen es espejo de un supra desnivel de ST posterior y la " $R$ " prominente, es el espejo de la onda "Q"14. La presencia en el ECG standard de 12 derivaciones, con depresión ST horizontal, " $\mathrm{R}$ ” prominente, relación $\mathrm{R} / \mathrm{S}>1,0$ en V2 y la combinación de la depresión ST con una onda " $\mathrm{T}$ " alta y simétrica puede ayudar al diagnóstico.

La oclusión transitoria de la arteria circunfleja proximal con un balón de angioplastía 
demostró que las derivadas posteriores (V7, V8 y V9) eran las que mostraban la mayor elevación del segmento $\mathrm{ST}^{15}$. Por lo tanto, la adición de las derivadas posteriores aumenta la posibilidad de diagnóstico; ubicando en el $5^{\circ}$ espacio intercostal a nivel de la línea axilar posterior izquierda a V7, en la línea medio escapular izquierda a V8 y V9 en relación con el borde de músculos paraespinales izquierdos, observado un SDST $>1 \mathrm{~mm}$, se logra un incremento de la sensibilidad de 32 a $57 \%$ y especificidad de $98 \%{ }^{16}$. Otros estudios indican que con un SDST $\geq 0,5 \mathrm{~mm}$ se logra la significancia estadística ${ }^{17}$, ya que la distancia al músculo miocárdico es mayor por ende el complejo puede ser de menor amplitud (Tablas $1,2$ y 3$)$.

\section{Conclusiones}

Si bien el infarto agudo al miocardio de pared posterior verdadero es de baja incidencia; es probable que más de una vez lo enfrentemos por lo cual es vital su correcto diagnóstico.

El método clásico de ECG de 12 derivaciones muchas veces no es capaz de evidenciar los cambios discretos del SDST, por lo que recomendamos frente a su sospecha observar las imágenes especulares en V1-V2 y utilizar las derivadas posteriores, V7-V8-V9. Al mismo tiempo se puede buscar la imagen especular observando V1-V2 en la imagen invertida del ECG clásico.

\section{Tabla 1. Pistas del ECG}

1. Depresión ST horizontal y " $R$ " prominente en V1-V2

2. Relación $\mathrm{R} / \mathrm{S}>1,0$ en $\mathrm{V} 2$

3. Combinación de la depresión ST + una onda "T" alta y simétrica

4. Utilización de las derivadas posteriores con SDST $\geq 0,5 \mathrm{~mm}$ en $\mathrm{V} 7-\mathrm{V} 8-\mathrm{V} 9$

5. Invertir el papel del registro del ECG estándar de 12 derivaciones, para observar la imagen especular del SDST en la primeras precordiales (Figura 7 A, B y C)

\section{Tabla 2. Diagnóstico diferencial de onda "R" alta en V1}

1. BCRD algunas veces se presenta como complejo monomórfico con " $R$ " alta

2. Miocardiopatía hipertrófica de predominio septal

3. Síndrome de Wolff-Parkinson-White

4. Hipertrofia del ventrículo derecho

5. Normal en jóvenes $R>S \circ R=S$

\section{Tabla 3. Diagnóstico diferencial de Infradesnivel} de ST en V1 y V2

1. Sobrecarga de ventrículo derecho durante TEP masivo

2. BCRD con alteraciones secundarias repolarización

3. Síndrome de Wolff-Parkinson-White con alteraciones secundarias de repolarización

4. Normal en jóvenes T (-) V1V2V3

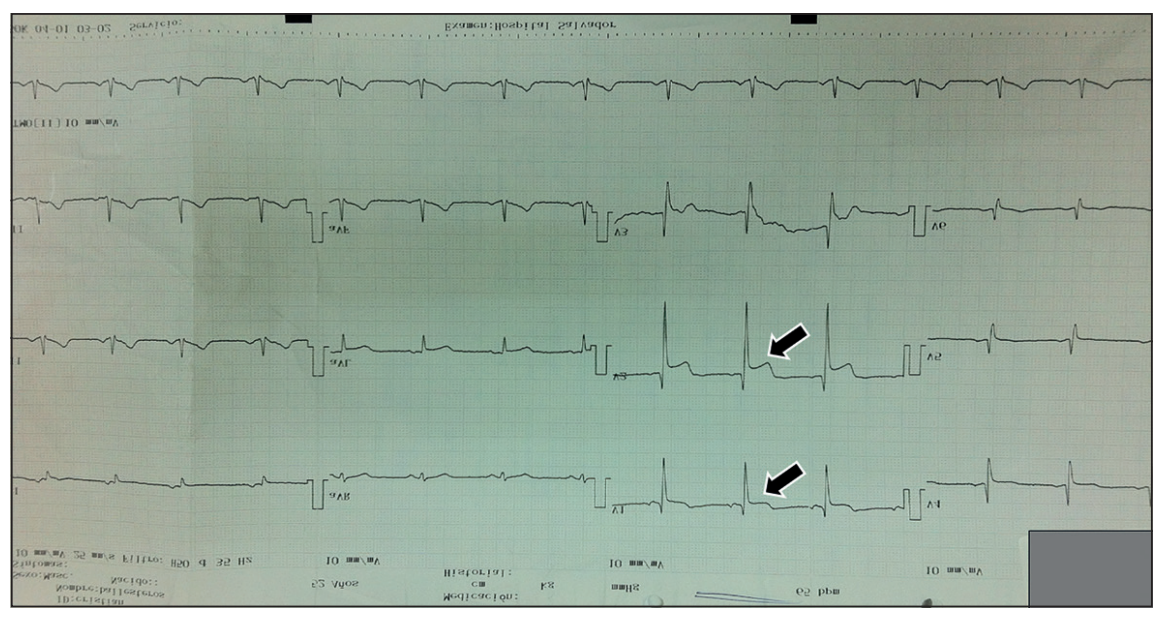

Figura 7A. Caso 1. 


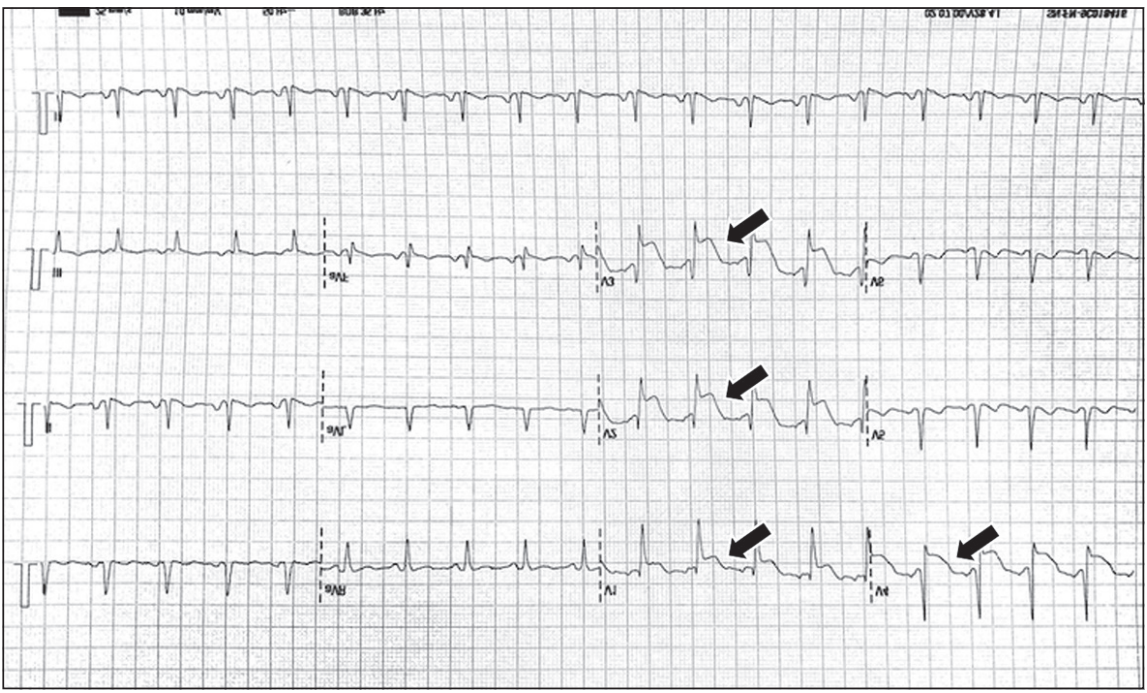

Figura 7B. Caso 2.

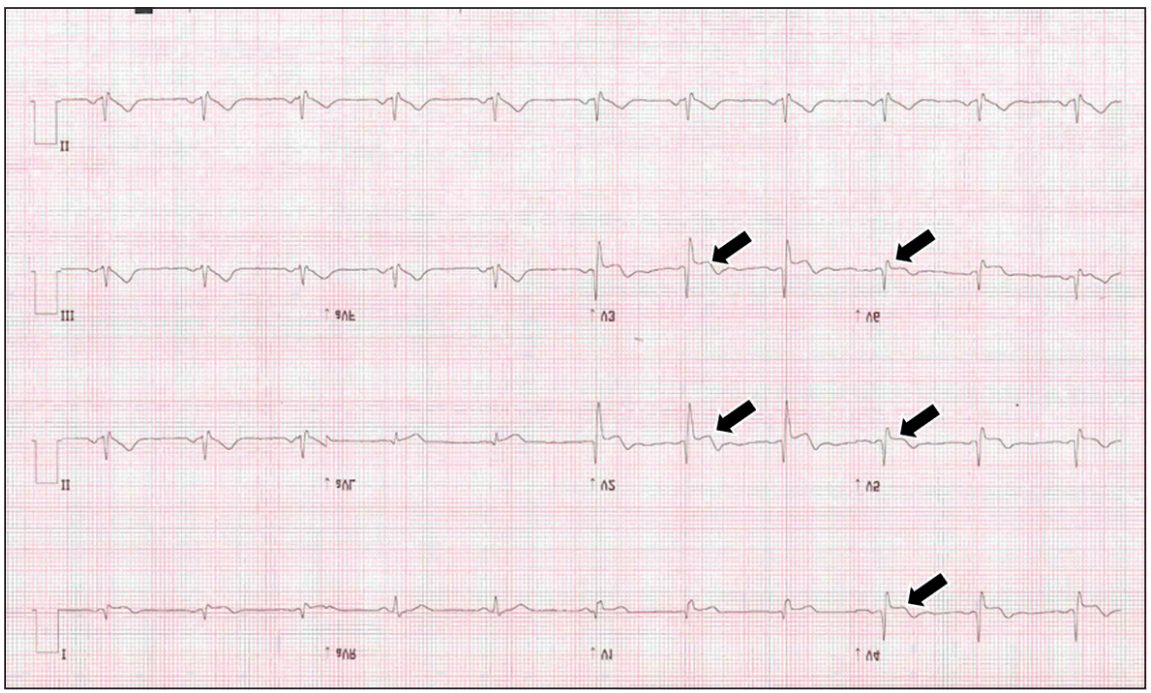

Figura 7C. Caso 3.

\section{Referencias}

1. Jneid H, Addison D, Bhatt D, Fonarow G, Gokak S, Grady K, et al. AHA/ACC Clinical Performance and Quality Measures for Adults With ST-Elevation and Non-ST-Elevation Myocardial Infarction. A Report of the American College of Cardiology/American Heart Association. Task Force on Performance Measures. JACC 2017; 70 (16): 2048-90.

2. Zalenski R, Rydman R, Sloan E, Hahn K, Cooke D, Fagan J, et al. Value of posterior and right ventricular leads in comparison to the standard 12-lead electro- cardiogram in evaluation of ST-segment elevation in suspected acute myocardial infarction. Am J Cardiol. 1997; 79 (12): 1579-85.

3. Oraii S, Maleki M, Tavakolian A, Eftekharzadeh M, Kamangar F, Mirhaji P. Prevalence and outcome of ST-segment elevation in posterior electrocardiographic leads during acute myocardial infarction. J Electrocardiol. 1999; 32 (3): 275-8.

4. Brady WJ, Erling B, Pollack M, Chan T. Electrocardiographic manifestations: acute posterior wall myocardial infarction. J Emerg Med. 2001; 20 (4): 391-401.

5. Arshad H, Ayaz S, Feurdean M. Comparison of revascu- 
larization and in-hospital mortality in patients with true posterior wall myocardial versus overall ST-Elevation myocardial infarction. JACC 2019; 73 (9): 249.

6. Perloff J. The recognition of strictly posterior myocardial infarction by conventional scalar electrocardiography. Circulation 1964; 30: 706-18.

7. Dunn WJ, Edwards JE, Pruitt RD. The electrocardiogram in infarction of the lateral wall of the left ventricle. A clinicopathological study. Circulation. 1956; (4 part 1): 540-55.

8. Bayés de Luna A. Nueva terminología de las paredes del corazón y nueva clasificación electrocardiográfica de los infartos con onda Q basada en la correlación con la resonancia magnética. Rev Esp Cardiol. 2007; 60 (7): 683-9.

9. Keeley EC, Boura JA, Grines CL. Primary angioplasty versus intravenous thrombolytic therapy for acute myocardial infarction: a quantitative review of 23 randomised trials. Lancet. 2003; 361 (9351): 13-20.

10. O'Gara PT, Kushner FG, Ascheim DD, Casey DE Jr, Chung MK, de Lemos JA, et al. 2013 ACCF/AHA guideline for the management of ST-elevation myocardial infarction: executive summary: a report of the American College of Cardiology Foundation/American Heart Association Task Force on Practice Guidelines: developed in collaboration with the American College of Emergency Physicians and Society for Cardiovascular Angiography and Interventions. Catheter Cardiovasc Interv. 2013; 82 (1): E1-27.
11. Matetzky S, Freimark D, Feinberg M, Novikov I, Rath S, Rabinowitz B, et al. Acute myocardial infarction with isolated ST-segment elevation in posterior chest leads V7-9: "hidden" ST-segment elevations revealing acute posterior infarction. J Am Coll Cardiol. 1999; 34 (3): 748-53

12. Moscucci M. Coronary angiography (capítulo 15) en Grossman \& Baim's. Catheterization, Angiography and Intervention, Philadelphia, United State of America. Lipincott, Williams and Wilkins; 8a Edition 2014; 295 334.

13. Das MK, Khan B, Jacob S, Kumar A, Mahenthiran J. Significance of a fragmented QRS complex versus a Q wave in patients with coronary artery disease. Circulation 2006; 113 (21): 2495-501.

14. Kawji M, Glancy DL. The value of reciprocal electrocardiographic leads. Am J Cardiol. 2017; 119 (9): 1490-91.

15. Aqel R, Hage F, Ellipeddi P, Blackmonn L, McElderry $\mathrm{H}$, Kay N, et al. Usefulness of three posterior chest leads for the detection of posterior wall acute myocardial infarction. Am J Cardiol. 2009; 103 (2): 159-64.

16. van Gorselen EOF, Verheugt FWA, Meursing BTJ, Oude Ophuis AJM. Posterior myocardial infarction: the dark side of the moon. Neth Heart J. 2007; 15 (1): 16-21.

17. Wung SF, Drew BJ. New electrocardiographic criteria for posterior wall acute myocardial ischemia validated by a percutaneous transluminal coronary angioplasty model of acute myocardial infarction. Am J Cardiol. 2001; 87 (8): 970-4. 